\title{
DNaseI Protects against Paraquat-Induced Acute Lung Injury and Pulmonary Fibrosis Mediated by Mitochondrial DNA
}

\author{
Guo Li, ${ }^{1}$ Li Yuzhen, ${ }^{2}$ Chen Yi, ${ }^{2}$ Chen Xiaoxiang, ${ }^{1}$ Zhou Wei, \\ Zhu Changqing, ${ }^{2}$ and Ye Shuang ${ }^{1}$ \\ ${ }^{1}$ Department of Rheumatology, South Campus, Ren Ji Hospital, School of Medicine, Shanghai Jiao Tong University, \\ Shanghai 200001, China \\ ${ }^{2}$ Department of Emergency Medicine, Ren Ji Hospital, School of Medicine, Shanghai Jiao Tong University, Shanghai 200001, China \\ Correspondence should be addressed to Zhu Changqing; zhucq1965@126.com and Ye Shuang; ye_shuang2000@163.com
}

Received 25 October 2014; Revised 21 December 2014; Accepted 23 December 2014

Academic Editor: Hartmut Jaeschke

Copyright (C) 2015 Guo Li et al. This is an open access article distributed under the Creative Commons Attribution License, which permits unrestricted use, distribution, and reproduction in any medium, provided the original work is properly cited.

\begin{abstract}
Background. Paraquat (PQ) poisoning is a lethal toxicological challenge that served as a disease model of acute lung injury and pulmonary fibrosis, but the mechanism is undetermined and no effective treatment has been discovered. Methods and Findings. We demonstrated that PQ injures mitochondria and leads to mtDNA release. The mtDNA mediated PBMC recruitment and stimulated the alveolar epithelial cell production of TGF- $\beta 1$ in vitro. The levels of mtDNA in circulation and bronchial alveolar lavage fluid (BALF) were elevated in a mouse of PQ-induced lung injury. DNaseI could protect PQ-induced lung injury and significantly improved survival. Acute lung injury markers, such as TNF $\alpha$, IL-1 $\beta$, and IL-6, and marker of fibrosis, collagen I, were downregulated in parallel with the elimination of mtDNA by DNaseI. These data indicate a possible mechanism for PQ-induced, mtDNA-mediated lung injury, which may be shared by other causes of lung injury, as suggested by the same protective effect of DNaseI in bleomycininduced lung injury model. Interestingly, increased mtDNA in the BALF of patients with amyopathic dermatomyositis-interstitial lung disease can be appreciated. Conclusions. DNaseI targeting mtDNA may be a promising approach for the treatment of PQinduced acute lung injury and pulmonary fibrosis that merits fast tracking through clinical trials.
\end{abstract}

\section{Introduction}

Acute lung injury and subsequent pulmonary fibrosis are a common clinically critical problem with extremely high mortality and morbidity. Multiple reasons, such as environmental factors, infections, and autoimmune factors, can trigger or perpetuate this pathophysiological process of aberrant lung injury and healing, but its precise mechanism is largely undetermined. Currently, no effective treatment has been shown to halt or reverse its development or progression [1].

Paraquat (1,1-dimethyl-4,4-bipyridinium dichloride, PQ) is one of the most widely used nonselective bipyridyl herbicides around the world, particularly in developing countries, such as China and India. PQ poisoning is a lethal toxicological challenge to humans and animals and is characterized by acute lung injury and irreversible pulmonary fibrosis [25]. No known antidote for PQ has been discovered, and the treatment options are merely supportive [6]. Thus, exploring the mechanism of PQ-induced lung injury may help to develop life-saving treatments for PQ poisoning and serve as a unique disease model for studying other types of acute lung injury and pulmonary fibrosis.

It has been demonstrated that PQ is toxic to green plants by attacking chloroplasts and interfering with vital photosynthesis [7]. In mammals, mitochondria are the counterpart organelle to chloroplasts. PQ cytotoxicity as a result of targeting mitochondria was proposed and demonstrated both in vitro and in vivo several decades ago [8-10], but the exact mechanism of PQ-induced mitochondrial dysfunction and, more importantly, how this links to lung injury and fibrosis are unknown. It is noteworthy that PQ cannot be detected in the plasma a few hours after ingestion due to rapid clearance by the kidneys [11]. Therefore, a PQ-triggered but self-sufficient pathway that mediates the subsequent lung injury and fibrosis is likely. Mitochondria are endosymbionts 
TABLE 1: Primers used in the experiment.

\begin{tabular}{|c|c|c|}
\hline Genes & Forward primer & Reverse primer \\
\hline GAPDH (human) & $5^{\prime}$-GCACCGTCAAGGCTGAGAAC-3' & 5'-ATGGTGGTGAAGACGCCAGT-3' $^{\prime}$ \\
\hline Cytochrome B (human) & 5'-ATGACCCCAATACGCAAAAT-3' & $5^{\prime}$-CGAAGTTTCATCATGCGGAG-3' \\
\hline$\alpha$-SMA (human) & 5'-GCGTGGCTATTCCTTCGTTACT-3' & 5'-GCTACATAACACAGTTTCTCCTTGATG-3' \\
\hline Vimentin (human) & $5^{\prime}$-CCGCCCTAGACGAACTGGGTC-3' & 5'-AGGCTGTGGACAGTGGCTTCTG-3' \\
\hline N-cadherin (human) & $5^{\prime}$-CCTCCAGAGTTTACTGCCATGAC-3' & $5^{\prime}$-GTAGGATCTCCGCCACTGATTC-3' \\
\hline E-cadherin (human) & $5^{\prime}$-GCCTCCTGAAAAGAGAGTGGAAG-3' & $5^{\prime}$-TGGCAGTGTCTCTCCAAATCCG-3' \\
\hline Tjp-1 (human) & $5^{\prime}$-GTCCAGAATCTCGGAAAAGTGCC-3' & $5^{\prime}$-CTTTCAGCGCACCATACCAACC-3' \\
\hline Cytokeratin (human) & 5'-AGCAGAATCGGAAGGACGCTGA-3' & 5'-ACCTCGCTCTTGCTGGACTGAA-3' \\
\hline$\beta$-actin (mice) & 5'-ATGCTCCCCGGGCTGTAT-3' & 5'-CATAGGAGTCCTTCTGACCCATT-3' \\
\hline Cytochrome B (mice) & 5'-CCTATCAGCCATCCCATAT-3' & $5^{\prime}$-GGAAGAGGAGGTGAACGA-3' \\
\hline
\end{tabular}

that originated from purple bacteria approximately $1.5 \times 10^{9}$ years ago [12] and are the only organelle of the mammalian cell except for the nucleus that contains its own DNA, that is, mitochondrial DNA (mtDNA) [13]. mtDNA contains a higher frequency of unmethylated cytosine-phosphateguanine $(\mathrm{CpG})$ dinucleotides, similar to bacterial DNA, which can promote innate immune responses through TLR-9 $[14,15]$. Circulating mtDNA from tissue injury was recently found to be essential to mediate the systemic inflammatory response and target organ damage [16]. Mitochondria have thus been termed the "Trojan Horse," capable of triggering inflammation and forming a vicious circle that results in profound tissue injury [17].

This study attempted to identify pathways underlying the role of PQ in mitochondrial dysfunction and mtDNA release in lung injury. The results may herald new interventions for treating this fatal toxicological condition. In addition, they may also suggest new methods for the management of other forms of acute lung injury and pulmonary fibrosis.

\section{Methods}

2.1. Reagents and Cell Lines. Paraquat and DNaseI were purchased from Sigma (St. Louis, MO, USA). Human alveolar type II-like epithelial A549 cells, human pulmonary fibroblast (HFL1) cells, and human pulmonary artery endothelial cells (HPAECs) were obtained from Cell Bank (Shanghai Institute of Cell Biology, China). The culture conditions were adapted from a previous report [18]. The Cell Counting Kit-8 (Dojindo, Japan), the mitochondrial-specific cationic dye JC1 (Molecular Probes, OR, USA), human IL-1 $\beta$, IL-6, TNF- $\alpha$, and TGF- $\beta 1$ ELISA kits (R\&D, Minneapolis, MN, USA), and the Vascular Permeability Kit (Millipore Corporation) were all used according to the manufacturers' protocols.

2.2. Measurement of mtDNA and Real-Time PCR. A549 cells were seeded into sterile, flat-bottom, 6 -well plates at $0.5 \times$ $10^{6}$ cells/well and grown overnight to $80 \%$ confluence. They were challenged with a control or various concentrations of PQ. Cell supernatants were harvested and centrifuged at $800 \mathrm{rpm}$ for $5 \mathrm{~min}$ to remove cellular debris. mtDNA was extracted from the supernatants using a QIAamp DNA Blood Mini Kit (Qiagen, USA). The concentration of mtDNA was determined using a standard curve generated by quantitative PCR (qPCR) (construct plasmids (PGM$\mathrm{T}$ ) containing human or mouse mitochondrial CytoB gene sequences; the primers are provided in Table 1). Total RNA was extracted using TRIzol reagent (Invitrogen, USA) and reverse-transcribed using the Prime Script cDNA Synthesis Kit (Takara, Japan). The primers for $\alpha$-SMA, type I collagen, type III collagen, vimentin, N-cadherin, E-cadherin, TJP-1, cytokeratin, and $\beta$-actin are also provided in Table 1. qPCR was performed using SYBR Premix Ex (Takara, Japan) on an ABI Prism 7900HT system.

2.3. Chemotaxis Assays and Flow Cytometry. PQ-primed A549 cell supernatants enriched for mtDNA (approximately $10^{4}$ copies $/ \mu \mathrm{L}$ ) were treated with or without DNaseI for the chemotaxis assay. Whole blood samples were obtained from healthy volunteers. Cells were isolated immediately by a one-step gradient centrifugation method using Polymorphprep reagent according to the described protocol [19]. The chemotactic responses of PBMCs and PMNs were assessed by transwell cell culture chambers with polycarbonate filters with $5 \mu \mathrm{m}$ pores. The fold chemotaxis index was calculated by dividing the number of cells migrating in the presence of supernatants by those migrating toward medium alone. Anti-CD14-APC, anti-CD3-FITC, and anti-CD19-PE (BD Biosciences, San Jose, CA) were used to distinguish the subtypes of the migrated cells by flow cytometry.

2.4. Real-Time Cell Analysis. HPAECs or HFL1 cells were seeded into 96-well microtiter plates (E plate) at a density of 5000 cells/well. After cell synchronization, the cells were treated with different stimuli and monitored. Transendothelial monolayer electrical resistance [20] and the cell index were measured using the xCELLigence Real-Time Cell Analyzer (Roche, USA).

2.5. Animals and Study Protocols. C57BL/6J mice (8-10 weeks of age, $n=144$ ), all males, were purchased from the Shanghai SLAC Laboratory Animal Co. Ltd. (Shanghai, China). The mice were intraperitoneally injected with PQ $(40 \mathrm{mg} / \mathrm{kg}$ or $25 \mathrm{mg} / \mathrm{kg}$, as indicated) or normal saline on day 1 . Some mice were injected intravenously with DNaseI $(0.3 \mathrm{mg} / \mathrm{kg}$, $3 \mathrm{mg} / \mathrm{kg}$, or $30 \mathrm{mg} / \mathrm{kg}$ ) or vehicle on day 0 (one day before the PQ or sham exposure), day 2, day 5, and day 8 . Before 


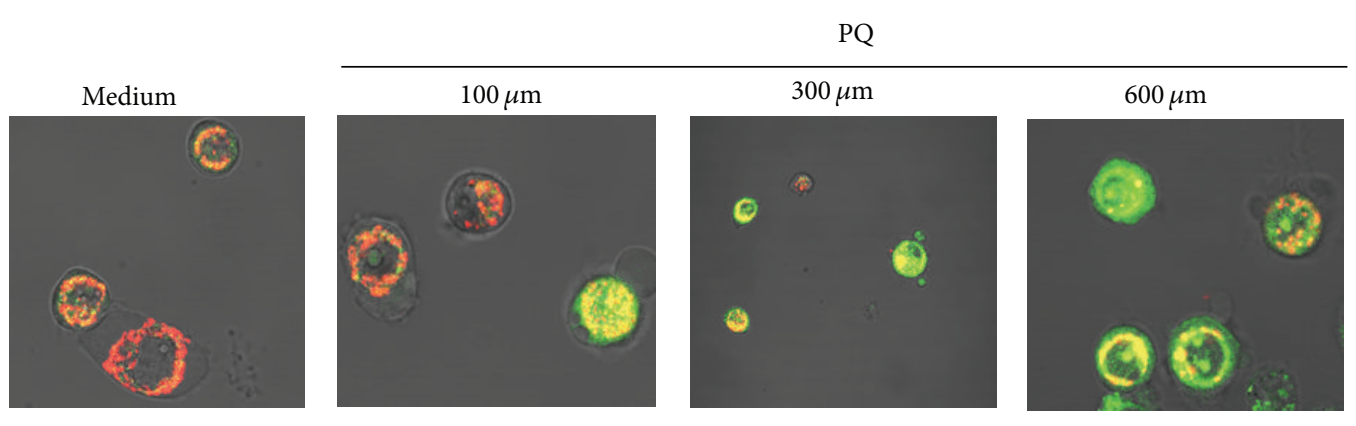

(a)

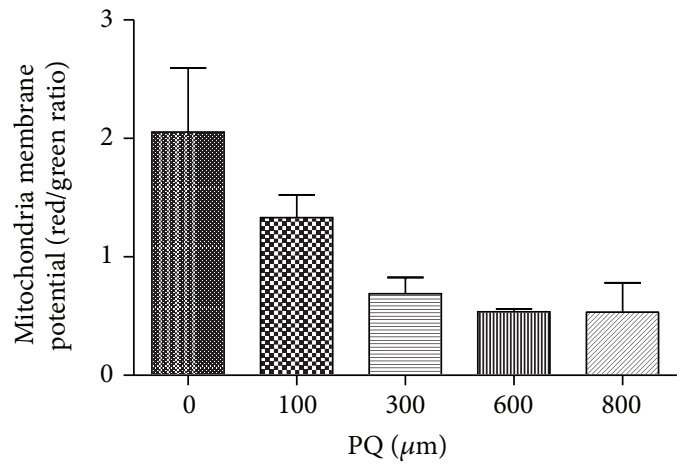

(b)

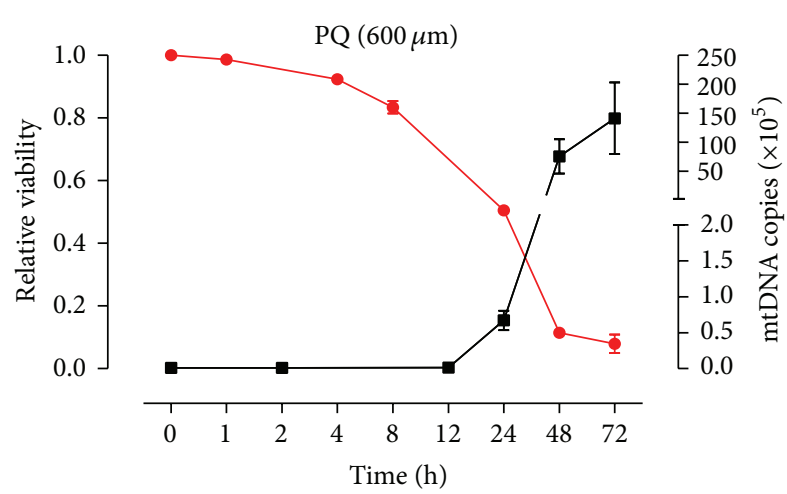

(d)

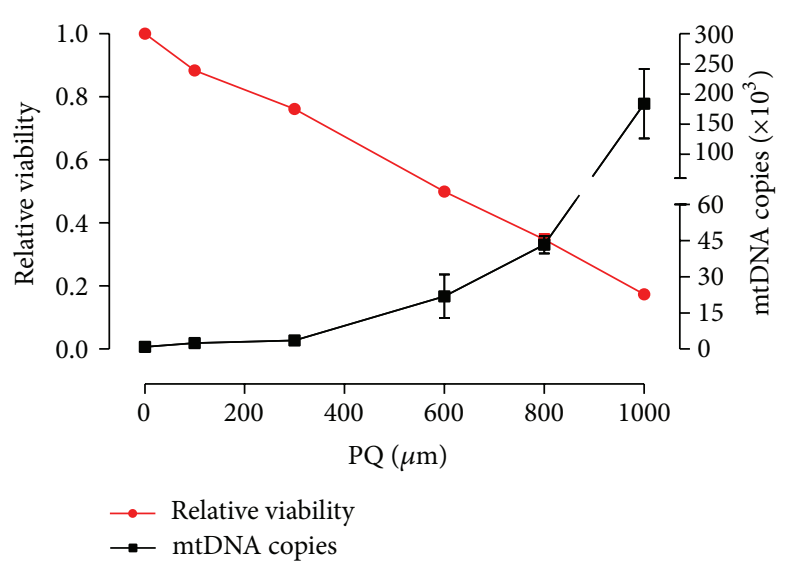

(c)

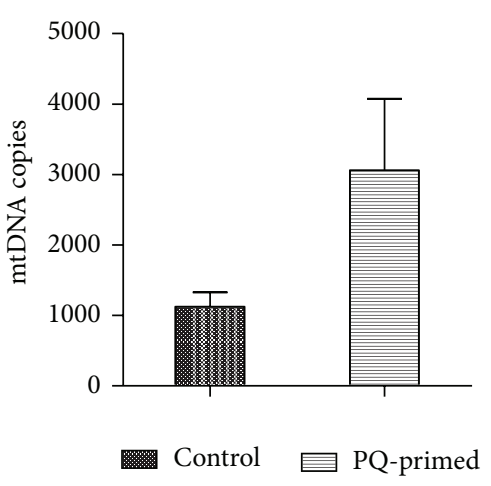

(e)

FIgURE 1: PQ injures mitochondria and leads to mtDNA release. ((a)-(b)) The mitochondrial membrane potential decreased in A549 cells (changed from red to green) following a $24 \mathrm{~h}$ exposure to various doses of PQ. PQ reduced A549 cell viability (red line) in a dose- (c) and time-dependent manner ((d), PQ $600 \mu \mathrm{m})$ and was correlated with increased mtDNA release (black line) into the supernatant. (e) A549 cells were incubated with or without (control) $600 \mu \mathrm{m}$ PQ for $12 \mathrm{~h}$ and then washed. Fresh medium was added, and the cells were cultured for $12 \mathrm{~h}$. The supernatant mtDNA level was elevated among PQ-primed cells.

euthanasia, the mice were anesthetized with pentobarbital $(60 \mathrm{mg} / \mathrm{kg})$ and subsequently underwent a median thoracosternotomy, bronchoalveolar lavage, exsanguination via the inferior vena cava, and removal of both lungs. The left lung was cut into two pieces, snap-frozen in liquid nitrogen, and stored at $-80^{\circ} \mathrm{C}$ until mRNA extraction. The right lung was immediately fixed in $10 \%$ neutral buffered formalin and embedded in paraffin for hematoxylin and eosin staining (HE) or Masson's trichrome staining. Survival experiments (for each group, $n=10$ ) were repeated three times. Survival curves were calculated by pooling the data of the three independent experiments. The bleomycin-induced lung injury model was generated according to a previous report [21]. Survival experiment was also carried out accordingly. A quantitative fibrosis scale (Ashcroft scale) was used [22]. Immunohistochemical staining for $\alpha$-SMA was performed according to the manufacturer's instructions. The study protocols were approved by the Animal Care Committees of Shanghai Jiao Tong University School of Medicine.

2.6. BALF from Patients with Amyopathic DermatomyositisRelated Interstitial Lung Disease (CADM-ILD) or Controls. 


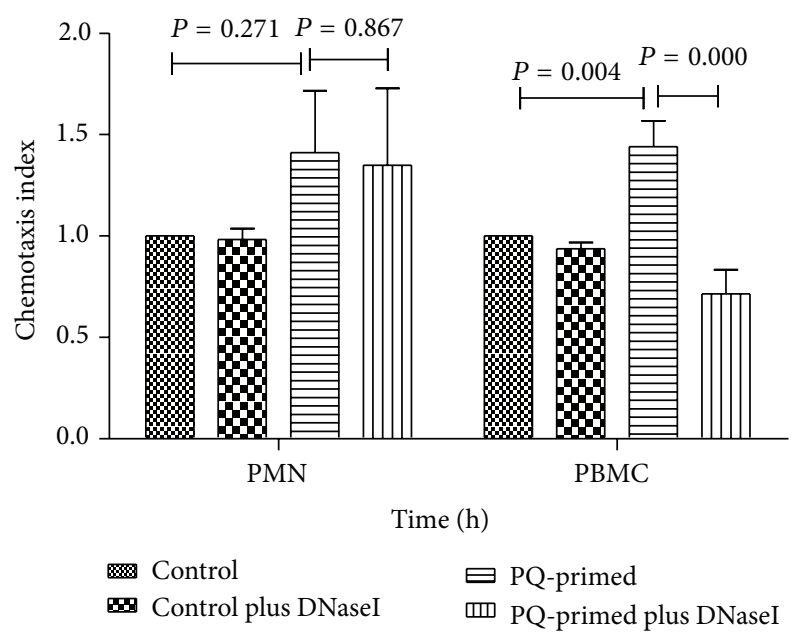

(a)

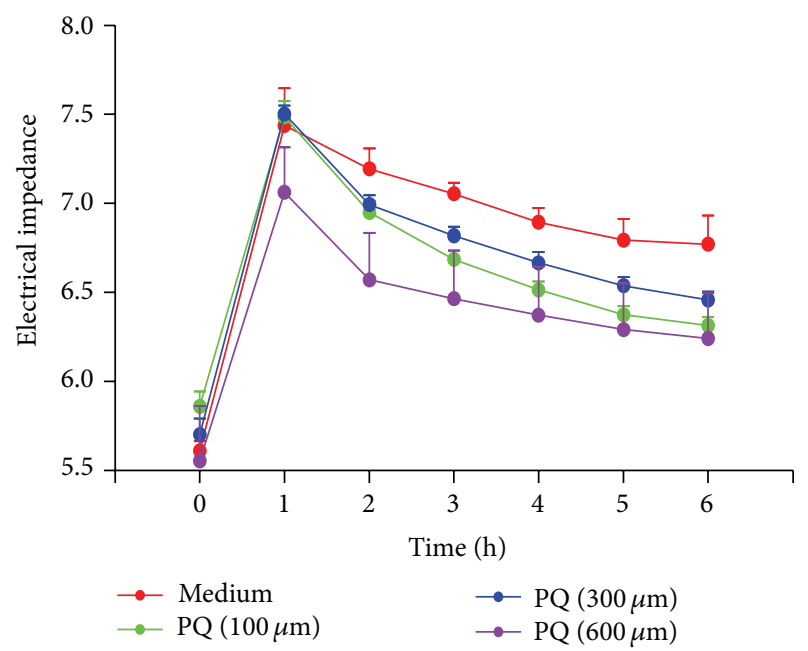

(c)

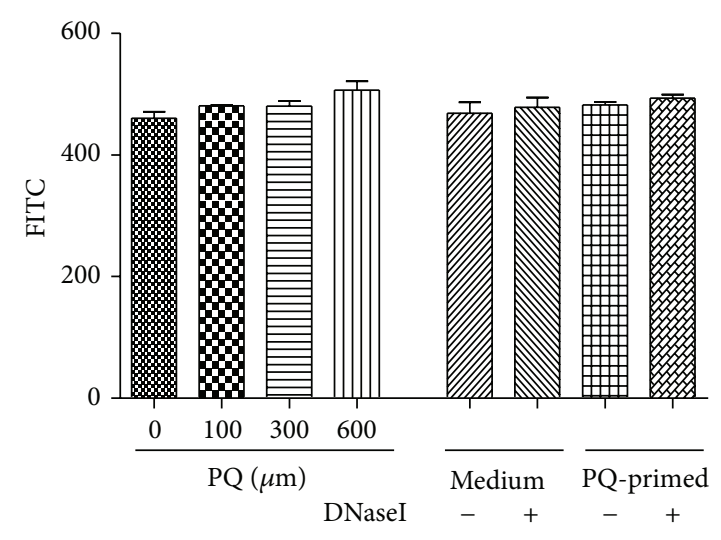

(b)

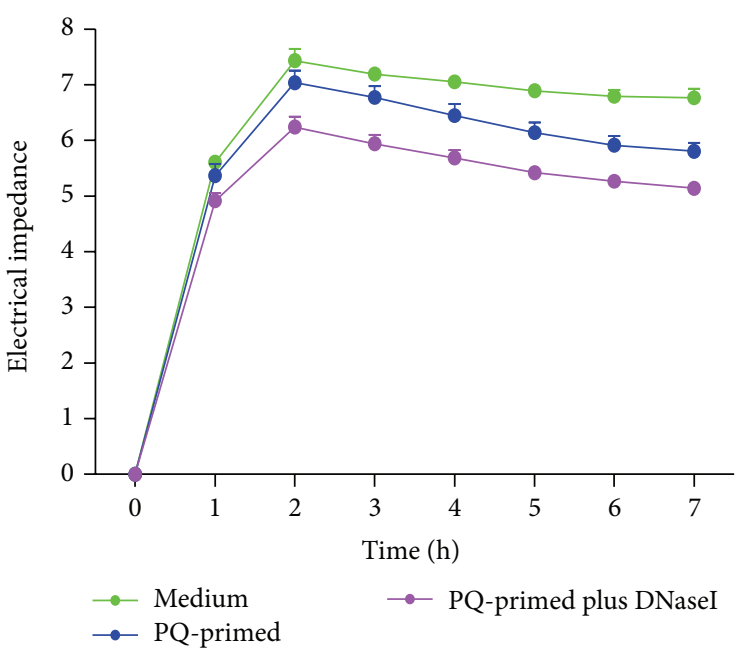

(d)

FIgURE 2: PQ-induced mtDNA release can enhance PBMC recruitment. (a) The chemotactic index of PBMCs but not PMNs increased after incubation with PQ-primed mtDNA-enriched supernatant. (b) The endothelial transwell fluorescein leakage assay displayed no difference in the presence of either PQ or PQ-primed mtDNA. ((c)-(d)) PQ and PQ-primed mtDNA had no statistical significant impact on endothelial cell integrity, as assessed with transendothelial monolayer electrical resistance.

BALF was obtained from patients with CADM-ILD $(n=14)$ or patients with pulmonary solitary mass or nodule $(n=$ 11) as controls. All patients were following a standardized bronchoscope procedure, and controls' BALF was recovered from the contralateral ("normal") side of the middle lobe. The study protocol was approved by the institutional review board of Shanghai Renji Hospital, Shanghai Jiao Tong University School of Medicine, with patients' informed consent obtained.

2.7. Statistical Analysis. The data are shown as the mean \pm SEM of at least three independent experiments. The statistical significance between groups was analyzed using GraphPad Prism v4.0 Software (San Diego, CA, USA). Twogroup comparisons of continuous data were assessed by either a two-tailed Student's $t$-test or a nonparametric MannWhitney $U$ test. ANOVA with a Bonferroni correction was used for multiple comparisons. Kaplan-Meier survival curves were calculated. A $P$ value less than 0.05 was considered significant.

\section{Results}

3.1. PQ Injures Mitochondria and Leads to mtDNA Release. We first assessed the impact of PQ on mitochondria in human alveolar type II-like epithelial A549 cells by fluorescence staining with the mitochondrial membrane potentialdependent dye JC-1. The mitochondrial membrane potential was decreased in the presence of PQ in a concentrationdependent manner (Figures 1(a) and 1(b)), indicating that the integrity of the mitochondria was disrupted by PQ. A similar result was obtained in a CCK8 assay, which demonstrated a reduction of cell viability and increased mtDNA release into the cell supernatant in a PQ dose- and time-dependent manner (Figures $1(\mathrm{c})$ and $1(\mathrm{~d})$ ). To further mimic the rapid clearance of PQ in vivo, cells were treated with PQ for $12 \mathrm{~h}$ 


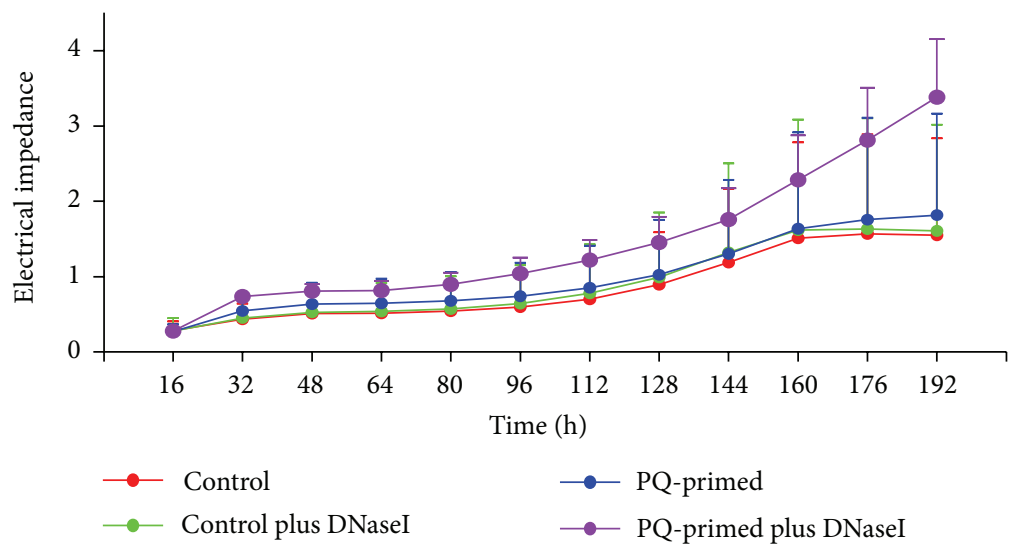

(a)

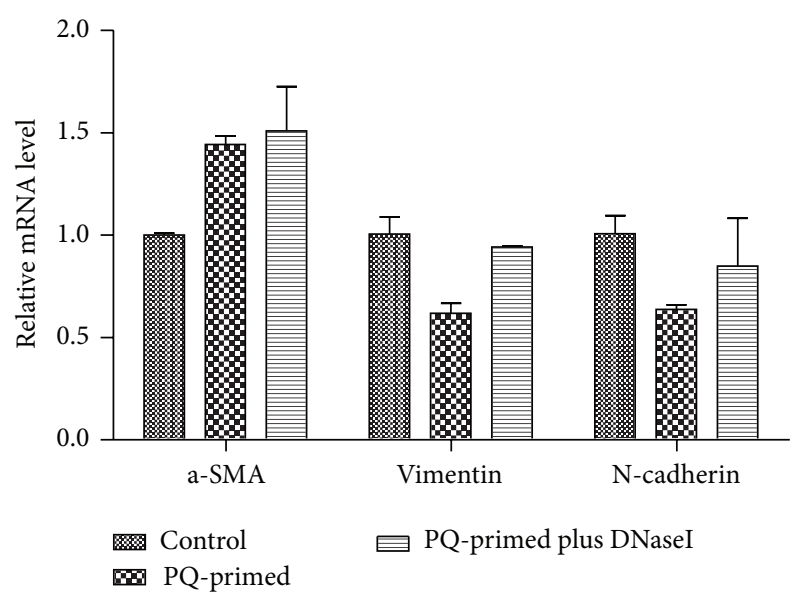

(c)

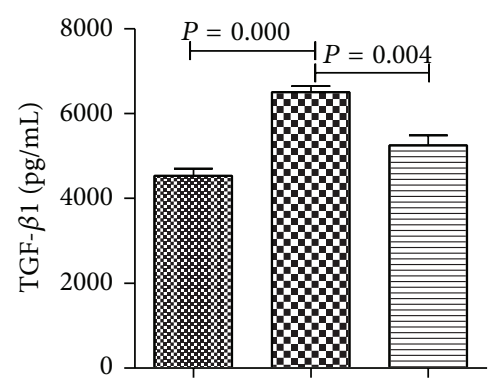

(b)

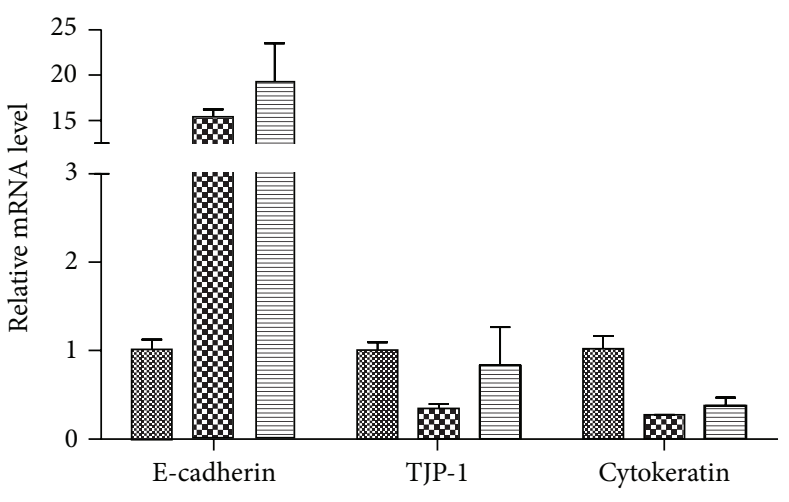

(d)

FIGURE 3: PQ-primed mtDNA stimulates alveolar epithelial (A549) cell production of TGF- $\beta 1$. (a) PQ-primed mtDNA did not stimulate fibroblast (HFL1) proliferation. (b) PQ-primed mtDNA increased A549 cell TGF- $\beta 1$ expression but had no effect on epithelial-mesenchymal transition marker expression (c) nor on downregulating epithelial marker expression (d).

(PQ-primed) and then washed. Fresh medium was added, and the cells were incubated for $12 \mathrm{~h}$. mtDNA release into the supernatant continued after PQ removal (Figure 1(e)), which is consistent with an ongoing injury response. PQprimed supernatants enriched with $\mathrm{mtDNA}$ were used in the following secondary cultures.

\subsection{PQ-Induced mtDNA Release Can Enhance PBMC Recruit-} ment. PQ-induced acute lung injury is characterized by inflammatory responses. We therefore investigated whether PQ-primed mtDNA could recruit effector cells. Indeed, enhanced chemotaxis of PBMCs but not neutrophils was observed under PQ-primed mtDNA-enriched conditions in a transwell assay; the effect was abolished when DNaseI was added to remove the mtDNA (Figure 2(a)). Flow cytometry analysis showed no difference in chemotaxis between PBMC subtypes, including T cells, B cells, NK cells, and monocytes (data not shown). This is likely to be an active attraction process instead of passive leaking due to the breakdown of the endothelial barrier. There was no significant change in transendothelial permeability as measured using transendothelial monolayer electrical resistance and an endothelial transwell fluorescein leakage assay, regardless of the presence of PQ or PQ-primed mtDNA-containing supernatants (Figures 2(b), 2(c), and 2(d)).

3.3. mtDNA Stimulates Alveolar Epithelial Cell Production of TGF- $\beta 1$. We next evaluated how PQ-induced pulmonary fibrosis was mediated by mtDNA. We initially investigated human pulmonary fibroblast (HFL1) cells and observed no direct proproliferation effect of PQ-primed mtDNA on the fibroblasts (Figure 3(a)). It was also possible that the recruited circulating monocytes could be precursors that transform into fibrocytes [23]. However, the impact of this transformation could not be observed in the presence of mtDNA or $\mathrm{PQ}$ in a conditioned culture of mouse splenocytes (data not shown). Epithelial-mesenchymal transition may also occur and be essential in pulmonary fibrosis. Indeed, PQ-primed mtDNA stimulated alveolar epithelial cells (A549) to produce TGF- $\beta 1$ (Figure 3(b)), although the epithelial-mesenchymal transition markers $\alpha$-SMA, vimentin, and $\mathrm{N}$-cadherin were not increased. Likewise, real-time PCR demonstrated that the epithelial markers E-cadherin, TJP-1, and cytokeratin were not downregulated (Figures 3(c) and 3(d)). Quite opposite, 

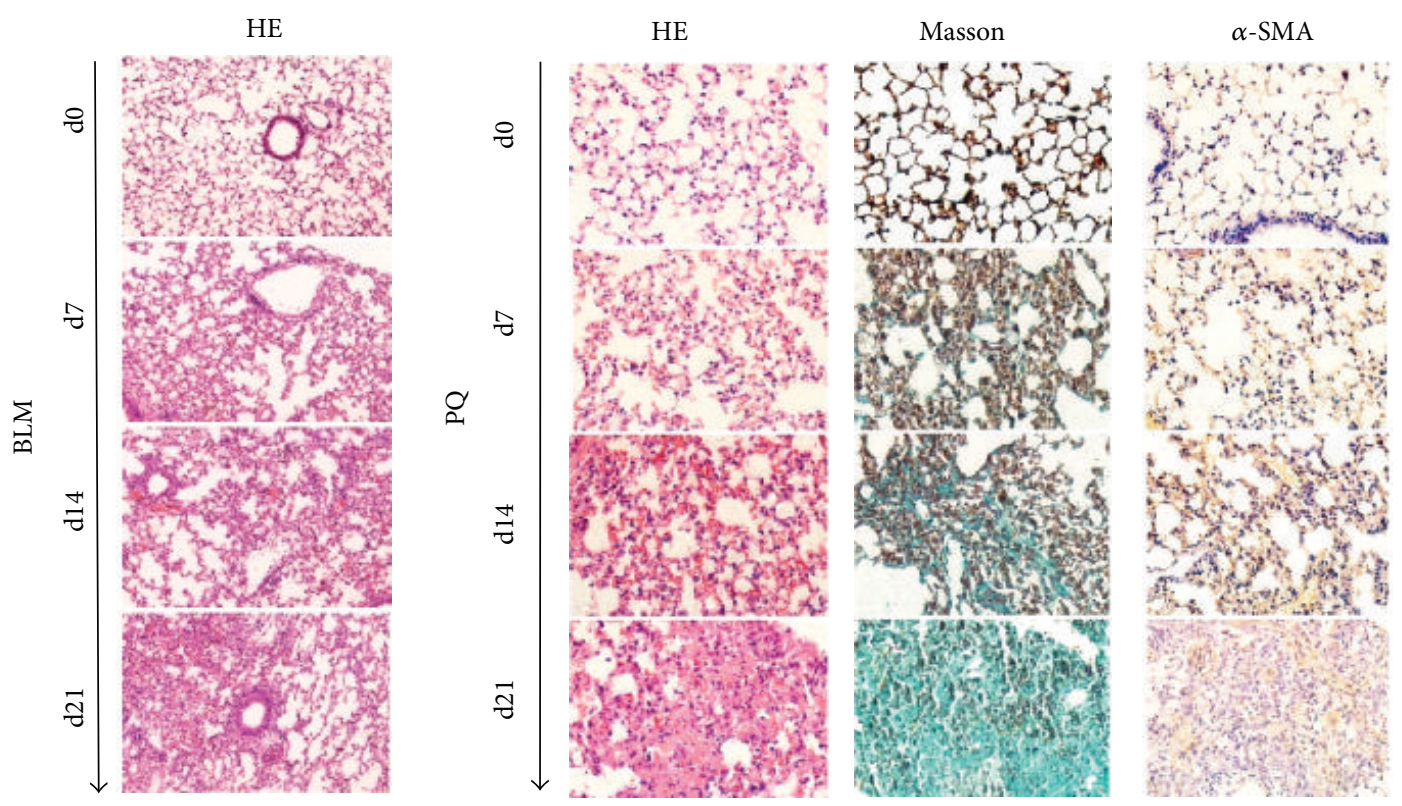

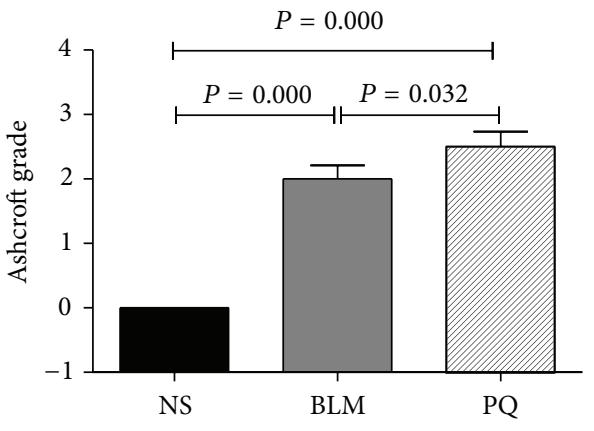

(b)

(a)

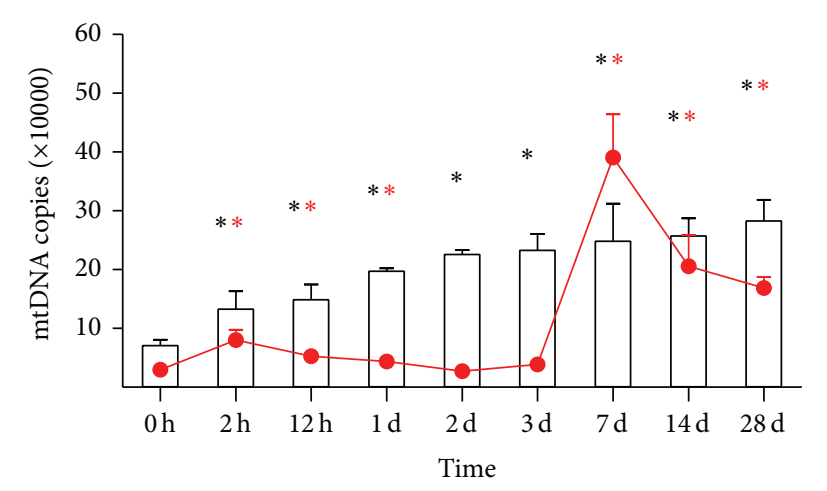

(c)

FIGURE 4: The levels of circulating and BALF mtDNA are elevated in a mouse model of PQ-induced lung injury. ((a)-(b)) C57BL/6 mice injected with PQ (25 mg/kg, i.p., d1 and d3) displayed typical acute lung injury and pulmonary fibrosis compatible with the classic bleomycin (BLM) model. (c) The time course of mtDNA detection differed in the plasma (bar) and BALF (red line).

E-cadherin was upregulated by PQ for unknown reason, and this effect is apparently independent of mtDNA. Nevertheless, it is conceivable that TGF- $\beta 1$ acts as a critical factor driving fibrosis and is upregulated by PQ-primed mtDNA to further act in the fibrogenic pathway in PQ-induced pulmonary fibrosis.

3.4. Circulating and BALF mtDNA Are Elevated in a Mouse Model of PQ-Induced Lung Injury. A PQ-induced lung injury model was established ( $25 \mathrm{mg} / \mathrm{kg}$ PQ intraperitoneally injected on day 1 and day 3). The model resulted in better homogeneity in lung pathology compared to the classic bleomycin (BLM) model, which is intratracheally delivered (Figures 4(a) and 4(b)). The mtDNA level was elevated in the plasma and BALF of mice administered PQ, but the patterns differed between the two (Figure 4(c)). The mtDNA level peaked in the BALF at day 7 , which is more representative of acute lung injury that subsequently induces pulmonary fibrosis.
3.5. DNaseI Protects PQ or Bleomycin-Induced Lung Injury in Mice and Improves Survival. DNaseI was administered intravenously 1 day prior to (day 0 ) exposure to the lethal dose of PQ (40 mg/kg, i.p.) and on days 2, 5, and 8 after the PQ challenge. DNaseI resulted in significant protection against PQ poisoning, as demonstrated by up to $70 \%$ survival observed in the DNaseI treatment group compared to the $100 \%$ mortality observed in the PQ control (Figure 5(a)). BALF and circulating mtDNA were eliminated in vivo by DNaseI (Figure 5(b)) in parallel with the downregulation of acute lung injury markers, including BALF total protein exudation, TNF $\alpha$, IL-1 $\beta$, and IL-6, at day 3 and marker of fibrosis, collagen I, at day 28 in a DNaseI dose-dependent manner (Figures 5(c) and 5(d)). The improved survival was also appreciated in the classic bleomycin-induced pulmonary fibrosis mice model. $90 \%$ survival was achieved in the DNaseI treatment groups versus a $50 \%$ survival in the bleomycin control group on day 28 (Figure 6(a)). 


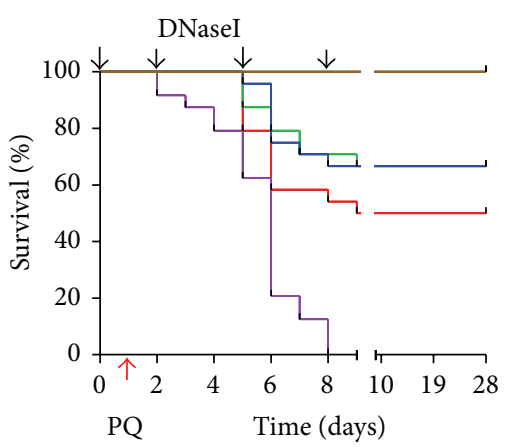

- DNaseI $0.3 \mathrm{mg} / \mathrm{kg}+\mathrm{PQ} 40 \mathrm{mg} / \mathrm{kg}$

- DNaseI $3 \mathrm{mg} / \mathrm{kg}+\mathrm{PQ} 40 \mathrm{mg} / \mathrm{kg}$

- DNaseI $30 \mathrm{mg} / \mathrm{kg}+\mathrm{PQ} 40 \mathrm{mg} / \mathrm{kg}$

- DNaseI $30 \mathrm{mg} / \mathrm{kg}+$ sham

- Vehicle + PQ $40 \mathrm{mg} / \mathrm{kg}$

- Vehicle + sham

(a)

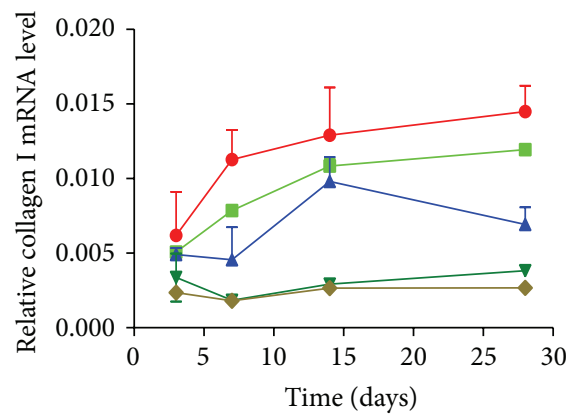

(c)
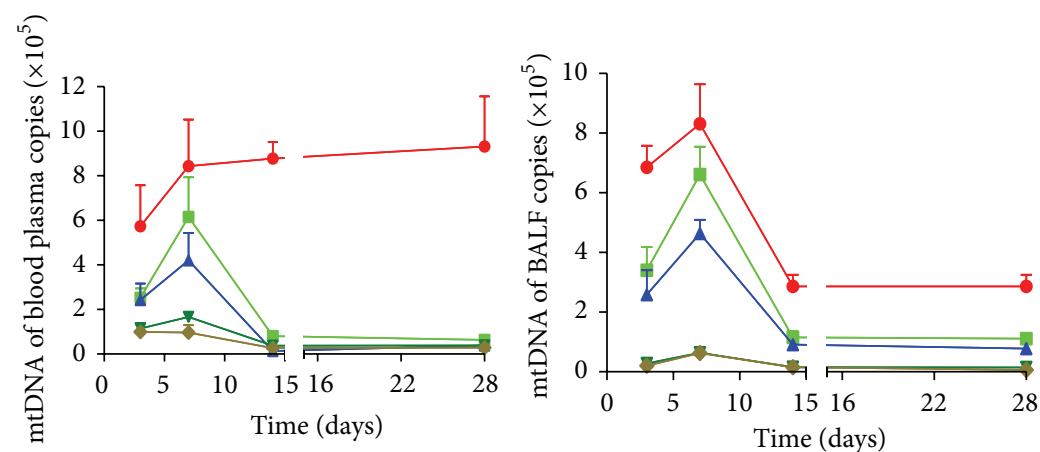

(b)
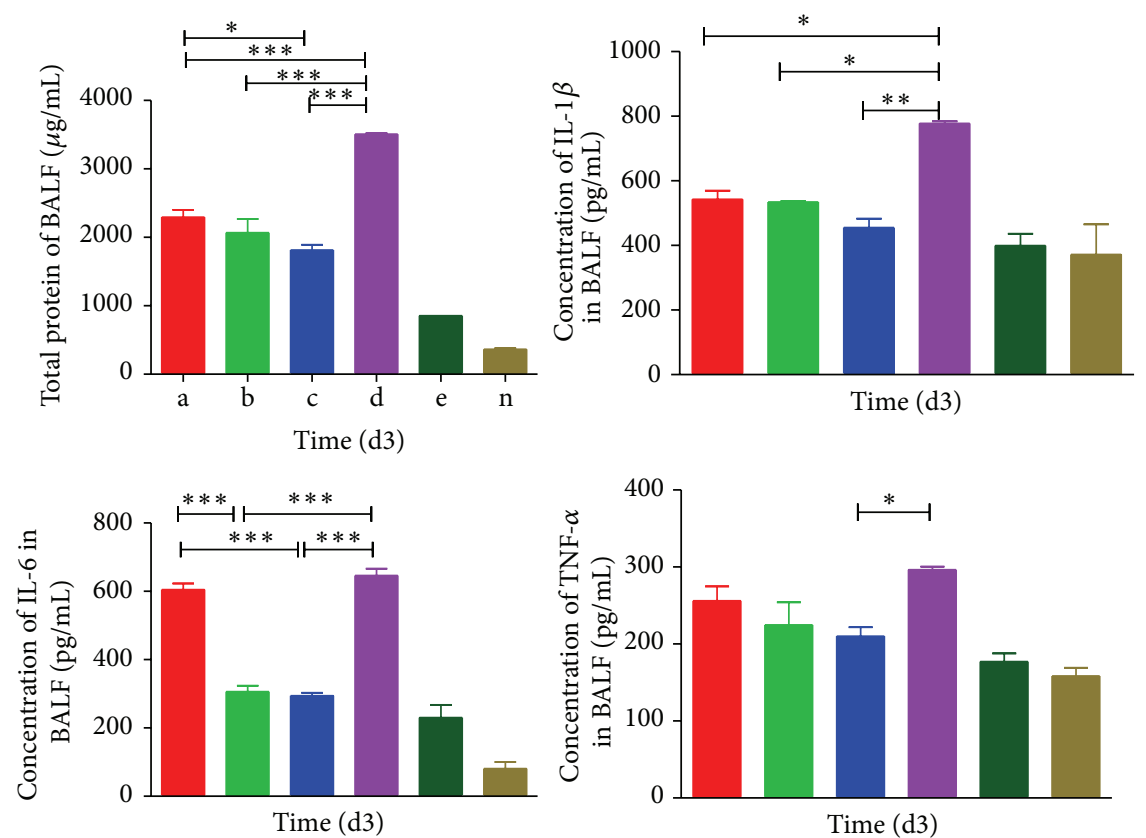

(d)

Figure 5: DNaseI prevents PQ-induced lung injury in mice and improves survival. (a) C57BL/6 mice ( $n=30$ for each group) were challenged with an acute lethal dose of PQ $(40 \mathrm{mg} / \mathrm{kg}$, i.p.) or sham exposure on day 1 . Some mice were administered DNaseI or vehicle at the indicated doses on days $0,2,5$, and 8 . (b) DNaseI suppressed the circulating and BALF mtDNA levels in a dose-dependent manner. ((c)-(d)) Downregulation of total protein exudation, TNF $\alpha$, IL-1 $\beta$, IL-6, and collagen I in a DNaseI dose-dependent manner.

3.6. mtDNA Were Increased in BALF from Patients with CADM-ILD. mtDNA in BALF from patients with CADMILD was significantly elevated compared to controls $(P=$ 0.0002) (Figure 6(b)).

\section{Discussion}

In the current study, we demonstrated that $\mathrm{PQ}$ injures mitochondria, causing subsequent mtDNA release. During PQ-induced oxidative stress, mitochondria can be a major source of reactive oxygen species (ROS) production [24-27]. $\mathrm{mtDNA}$, located close to the inner mitochondrial membrane where ROS are generated, is susceptible to oxidative damage [28]. In addition, ROS may also facilitate mtDNA release [29]. However, the mechanism by which PQ targets mitochondria is still undetermined and requires further investigation. As an example, Chen and colleagues observed that PQ-induced Nrf-2, an antioxidative transcriptional factor, in lung alveolar epithelial cells and Nrf-2 siRNA reversed the PQ-induced mRNA expression profile in vitro [30]. Paradoxically, in vivo data suggested that $\mathrm{Nrf2}^{-/-}$mice are more susceptible to gastric aspiration-induced acute lung injury [31], as well as to Staphylococcus aureus-induced lung injury [32]. Nevertheless, our data are the first to demonstrate that 


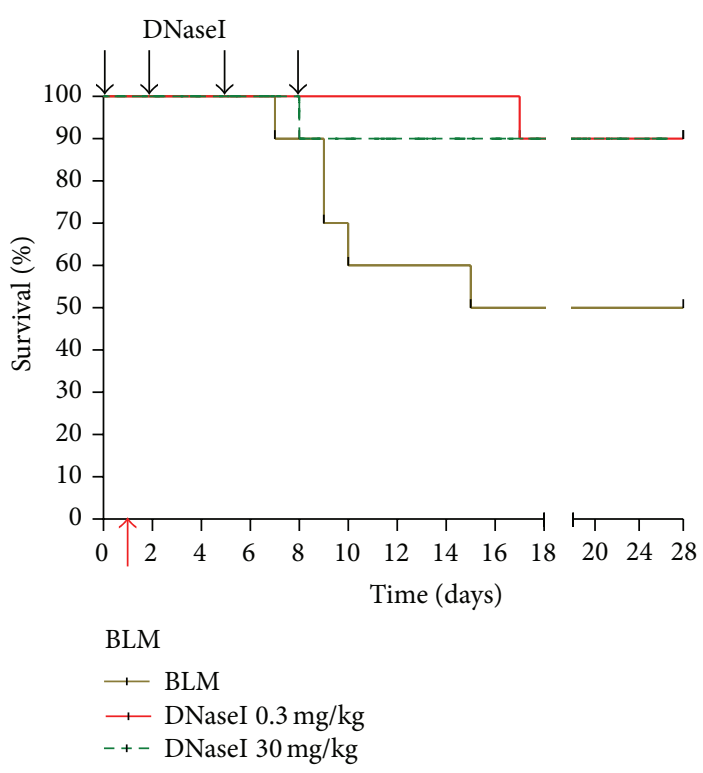

(a)

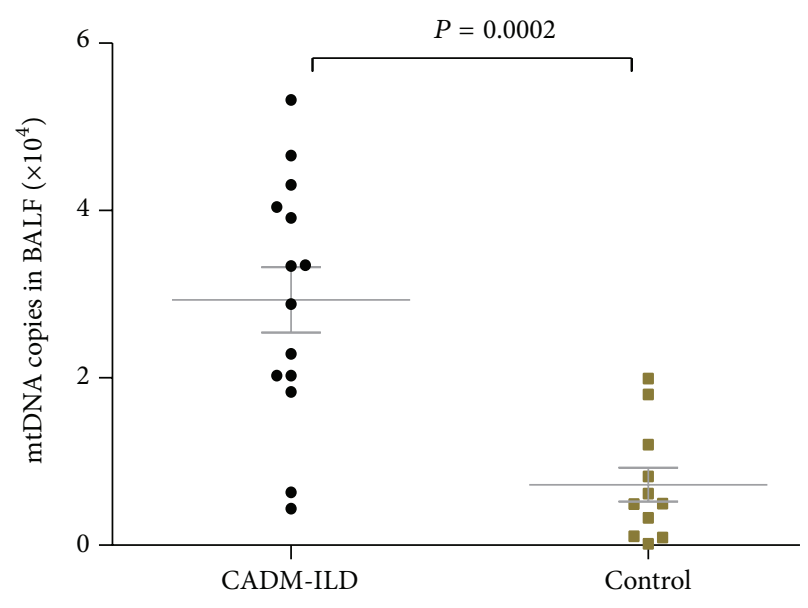

(b)

FIGURE 6: DNaseI improves survival in bleomycin-induced pulmonary fibrosis mice and mtDNA was increased in BALF from CADM-ILD patients. (a) Improved survival in bleomycin-induced pulmonary fibrosis mice by i.v. administration of DNaseI ( $n=10$ for each group). (b) mtDNA in BALF from patients with CADM-ILD $(n=14)$ was significantly elevated compared to controls $(n=11)$. Mean and SEM values were indicated.

mtDNA is the mediator of PQ-induced acute lung injury and pulmonary fibrosis. mtDNA is a double-stranded, closed circular molecule of 16,569 nucleotide pairs. As a potent innate immune system stimulator, Zhang and colleagues found that circulating mtDNA and formyl peptides can induce a systemic inflammatory response to injury via TLR9 and other DAMP pattern recognition pathways' activation [16]. According to our data, PQ-induced $\mathrm{mtDNA}$ is capable of mediating the recruitment of mononuclear cells but not polymorphonuclear cells. This chemotaxis process is apparently independent of endothelial barrier disruption. PQ-induced mtDNA can also enhance alveolar epithelial cell TGF- $\beta 1$ production. As one of the key profibrotic molecules, TGF- $\beta 1$ is elevated in the lung tissue of PQ treated mice according to our previous data [33], similar to the finding in bleomycin model [34]. More importantly, the effects of mtDNA in vitro are all reversible in the presence of DNaseI.

The most interesting finding was the in vivo observation that the intravenous administration of DNaseI displayed a striking protective effect against both PQ- and bleomycininduced lung injury in mice. It is noteworthy that early intervention with DNaseI in the acute lung injury phase is crucial and parallels the timing of the BALF mtDNA peak in PQ model. In addition to its wide use in molecular biology research, DNaseI has been approved as a nebulizing agent for patients with cystic fibrosis [35]. Endogenous DNaseI deficiency due to a genetic polymorphism in lupus has been postulated to be responsible for inappropriate nuclear debris clearance and results in immune system overload and antinuclear autoantibody generation [36, 37]. However, a phase I trial in SLE patients failed to display efficacy of the intravenous and subcutaneous administration of recombinant human DNaseI, although its safety profile was acceptable [38].

Caudrillier and colleagues recently found that the formation of neutrophil extracellular traps (NETs) is essential to mediate transfusion-related acute lung injury [39]. DNaseI and histone-blocking antibodies that inhibit NET formation are both protective against transfusion-related acute lung injury in mice. Although the pathogenesis of this acute lung injury is different from PQ-induced lung injury, a shared pathway may exist. For example, the possibility that mtDNA is an important NET component cannot be excluded. It is also informative that the mtDNA level is significantly elevated in the BALF of patients with CADM-ILD, which is another lethal autoimmune condition with acute lung injury and pulmonary fibrosis [40]. There are increasing evidences suggesting that mtDNA is a promising therapeutic target, such as mtDNA repair enzyme 8-oxoguanine DNA glycosylase 1 and DNA repair enzyme endonuclease III which all displayed a certain protective effect against different models of lung injury [41-44]. Taken together, the rationale for initiating clinical trials on DNaseI for PQ-induced lung injury is sound, and such attempts to tame the "Trojan Horse" in other forms of lung injury and pulmonary fibrosis will be breathtaking.

\section{Abbreviations \\ PQ: Paraquat mtDNA: Mitochondrial DNA.}

\section{Conflict of Interests}

The authors have declared that no conflict of interests exists. 


\section{Authors' Contribution}

Guo Li and Li Yuzhen contributed equally to this work.

\section{References}

[1] M. Cepkova and M. A. Matthay, "Pharmacotherapy of acute lung injury and the acute respiratory distress syndrome," Journal of Intensive Care Medicine, vol. 21, no. 3, pp. 119-143, 2006.

[2] R. E. Murray and J. E. Gibson, "A comparative study of paraquat intoxication in rats, guinea pigs and monkeys," Experimental and Molecular Pathology, vol. 17, no. 3, pp. 317-325, 1972.

[3] R. B. Bruyndonckx, A. I. Meulemans, M. B. Sabbe, A. A. Kumar, and H. H. Delooz, "Fatal intentional poisoning cases admitted to the University Hospitals of Leuven, Belgium from 1993 to 1996," European Journal of Emergency Medicine, vol. 9, no. 3, pp. 238-243, 2002.

[4] C.-M. Chen and A. C. Lua, "Lung toxicity of paraquat in the rat," Journal of Toxicology and Environmental Health A, vol. 60, no. 7, pp. 477-487, 2000.

[5] R. J. Dinis-Oliveira, J. A. Duarte, A. Sánchez-Navarro, F. Remião, M. L. Bastos, and F. Carvalho, "Paraquat poisonings: mechanisms of lung toxicity, clinical features, and treatment," Critical Reviews in Toxicology, vol. 38, no. 1, pp. 13-71, 2008.

[6] M. Ghazi-Khansari, G. Nasiri, and M. Honarjoo, "Decreasing the oxidant stress from paraquat in isolated perfused rat lung using captopril and niacin," Archives of Toxicology, vol. 79, no. 6, pp. 341-345, 2005.

[7] P. Slade, “The fate of paraquat applied to plants," Weed Research, vol. 6, no. 2, pp. 158-167, 1966.

[8] J. C. Gage, "The action of paraquat and diquat on the respiration of liver cell fractions," Biochemical Journal, vol. 109, no. 5, pp. 757-761, 1968.

[9] K. I. Hirai, H. Witschi, and M. G. Cote, "Mitochondrial injury of pulmonary alveolar epithelial cells in acute paraquat intoxication," Experimental and Molecular Pathology, vol. 43, no. 2, pp. 242-252, 1985.

[10] G. Y. Wang, K. Hirai, and H. Shimada, "Mitochondrial breakage induced by the herbicide paraquat in cultured human lung cells," Journal of Electron Microscopy (Tokyo), vol. 41, no. 3, pp. 181-184, 1992.

[11] E. D. Carson, "Fatal paraquat poisoning in Northern Ireland," Journal of the Forensic Science Society, vol. 12, no. 3, pp. 437-443, 1972.

[12] P. Costantini, V. Petronilli, R. Colonna, and P. Bernardi, "On the effects of paraquat on isolated mitochondria. Evidence that paraquat causes opening of the cyclosporin A-sensitive permeability transition pore synergistically with nitric oxide," Toxicology, vol. 99, no. 1-2, pp. 77-88, 1995.

[13] S. DiMauro and E. A. Schon, "Mitochondrial respiratory-chain diseases," The New England Journal of Medicine, vol. 348, no. 26, pp. 2656-2668, 2003.

[14] H. Hemmi, O. Takeuchi, T. Kawai et al., "A Toll-like receptor recognizes bacterial DNA," Nature, vol. 408, no. 6813, pp. 740$745,2000$.

[15] S. Bauer, C. J. Kirschning, H. Häcker et al., "Human TLR9 confers responsiveness to bacterial DNA via species-specific CpG motif recognition," Proceedings of the National Academy of Sciences of the United States of America, vol. 98, no. 16, pp. 9237-9242, 2001.
[16] Q. Zhang, M. Raoof, Y. Chen et al., "Circulating mitochondrial DAMPs cause inflammatory responses to injury," Nature, vol. 464, no. 7285, pp. 104-107, 2010.

[17] A. A. Manfredi and P. Rovere-Querini, "The mitochondriona Trojan horse that kicks off inflammation?" The New England Journal of Medicine, vol. 362, no. 22, pp. 2052-2134, 2010.

[18] P. Mitsopoulos and Z. E. Suntres, "Cytotoxicity and gene array analysis of alveolar epithelial A549 cells exposed to paraquat," Chemico-Biological Interactions, vol. 188, no. 3, pp. 427-436, 2010.

[19] Z. Fekete, C. J. Hauser, J. M. Adams et al., "Injury-enhanced calcium mobilization in circulating rat neutrophils models human PMN responses," Shock, vol. 16, no. 1, pp. 15-20, 2001.

[20] S. M. Camp, R. Bittman, E. T. Chiang et al., "Synthetic analogs of FTY720 [2-amino-2-(2-[4-octylphenyl]ethyl)-1,3-propanediol] differentially regulate pulmonary vascular permeability in vivo and in vitro," Journal of Pharmacology and Experimental Therapeutics, vol. 331, no. 1, pp. 54-64, 2009.

[21] N. Hattori, J. L. Degen, T. H. Sisson et al., "Bleomycin-induced pulmonary fibrosis in fibrinogen-null mice," Journal of Clinical Investigation, vol. 106, no. 11, pp. 1341-1350, 2000.

[22] T. Ashcroft, J. M. Simpson, and V. Timbrell, "Simple method of estimating severity of pulmonary fibrosis on a numerical scale," Journal of Clinical Pathology, vol. 41, no. 4, pp. 467-470, 1988.

[23] M. Niedermeier, B. Reich, M. R. Gomez et al., "CD4 ${ }^{+}$T cells control the differentiation of $\mathrm{Grl}^{+}$monocytes into fibrocytes," Proceedings of the National Academy of Sciences of the United States of America, vol. 106, no. 42, pp. 17892-17897, 2009.

[24] J. S. Bus and J. E. Gibson, "Paraquat: model for oxidant-initiated toxicity," Environmental Health Perspectives, vol. 55, pp. 37-46, 1984.

[25] V. Mollace, M. Iannone, C. Muscoli et al., "The role of oxidative stress in paraquat-induced neurotoxicity in rats: protection by non peptidyl superoxide dismutase mimetic," Neuroscience Letters, vol. 335, no. 3, pp. 163-166, 2003.

[26] A. Mohammadi-Bardbori and M. Ghazi-Khansari, "Alternative electron acceptors: proposed mechanism of paraquat mitochondrial toxicity," Environmental Toxicology and Pharmacology, vol. 26, no. 1, pp. 1-5, 2008.

[27] P. R. Castello, D. A. Drechsel, and M. Patel, "Mitochondria are a major source of paraquat-induced reactive oxygen species production in the brain," The Journal of Biological Chemistry, vol. 282, no. 19, pp. 14186-14193, 2007.

[28] H. van Remmen and A. Richardson, "Oxidative damage to mitochondria and aging," Experimental Gerontology, vol. 36, no. 7, pp. 957-968, 2001.

[29] K. Nakahira, J. A. Haspel, V. A. K. Rathinam et al., "Autophagy proteins regulate innate immune responses by inhibiting the release of mitochondrial DNA mediated by the NALP3 inflammasome," Nature Immunology, vol. 12, no. 3, pp. 222-230, 2011.

[30] Y.-W. Chen, Y.-T. Yang, D.-Z. Hung, C.-C. Su, and K.-L. Chen, "Paraquat induces lung alveolar epithelial cell apoptosis via Nrf2-regulated mitochondrial dysfunction and ER stress," Archives of Toxicology, vol. 86, no. 10, pp. 1547-1558, 2012.

[31] B. A. Davidson, R. R. Vethanayagam, M. J. Grimm et al., "NADPH oxidase and Nrf2 regulate gastric aspiration-induced inflammation and acute lung injury," The Journal of Immunology, vol. 190, no. 4, pp. 1714-1724, 2013.

[32] J. Athale, A. Ulrich, N. Chou MacGarvey et al., "Nrf2 promotes alveolar mitochondrial biogenesis and resolution of lung injury in Staphylococcus aureus pneumonia in mice," Free Radical Biology and Medicine, vol. 53, no. 8, pp. 1584-1594, 2012. 
[33] J. Qian, Y. Ye, L. Lv, C. Zhu, and S. Ye, "FTY720 attenuates paraquat-induced lung injury in mice," International Immunopharmacology, vol. 21, no. 2, pp. 426-431, 2014.

[34] T. Izumo, M. Kondo, and A. Nagai, "Effects of a leukotriene B4 receptor antagonist on bleomycin-induced pulmonary fibrosis," European Respiratory Journal, vol. 34, no. 6, pp. 1444-1451, 2009.

[35] H. J. Fuchs, D. S. Borowitz, D. H. Christiansen et al., "Effect of aerosolized recombinant human DNase on exacerbations of respiratory symptoms and on pulmonary function in patients with cystic fibrosis," The New England Journal of Medicine, vol. 331, no. 10, pp. 637-642, 1994.

[36] M. Napirei, H. Karsunky, B. Zevnik, H. Stephan, H. G. Mannherz, and T. Möröy, "Features of systemic lupus erythematosus in Dnasel-deficient mice," Nature Genetics, vol. 25, no. 2, pp. 177-181, 2000.

[37] K. Yasutomo, T. Horiuchi, S. Kagami et al., "Mutation of DNASE1 in people with systemic lupus erythematosus," Nature Genetics, vol. 28, no. 4, pp. 313-314, 2001.

[38] J. C. Davis Jr., S. Manzi, C. Yarboro et al., "Recombinant human Dnase I (rhDNase) in patients with lupus nephritis," Lupus, vol. 8, no. 1, pp. 68-76, 1999.

[39] A. Caudrillier, K. Kessenbrock, B. M. Gilliss et al., "Platelets induce neutrophil extracellular traps in transfusion-related acute lung injury," Journal of Clinical Investigation, vol. 122, no. 7, pp. 2661-2671, 2012.

[40] S. Ye, X. X. Chen, X. Y. Lu et al., "Adult clinically amyopathic dermatomyositis with rapid progressive interstitial lung disease: a retrospective cohort study," Clinical Rheumatology, vol. 26, no. 10, pp. 1647-1654, 2007.

[41] M. Hashizume, M. Mouner, J. M. Chouteau et al., "Mitochondrial-targeted DNA repair enzyme 8-oxoguanine DNA glycosylase 1 protects against ventilator-induced lung injury in intact mice," The American Journal of Physiology — Lung Cellular and Molecular Physiology, vol. 304, no. 4, pp. L287-L297, 2013.

[42] S. A. Gebb, A. Decoux, A. Waggoner, G. L. Wilson, and M. N. Gillespie, "Mitochondrial DNA damage mediates hyperoxic dysmorphogenesis in rat fetal lung explants," Neonatology, vol. 103, no. 2, pp. 91-97, 2013.

[43] J. M. Chouteau, B. Obiako, O. M. Gorodnya et al., "Mitochondrial DNA integrity may be a determinant of endothelial barrier properties in oxidant-challenged rat lungs," The American Journal of Physiology - Lung Cellular and Molecular Physiology, vol. 301, no. 6, pp. L892-L898, 2011.

[44] M. V. Ruchko, O. M. Gorodnya, A. Zuleta, V. M. Pastukh, and M. N. Gillespie, "The DNA glycosylase Oggl defends against oxidant-induced mtDNA damage and apoptosis in pulmonary artery endothelial cells," Free Radical Biology and Medicine, vol. 50, no. 9, pp. 1107-1113, 2011. 

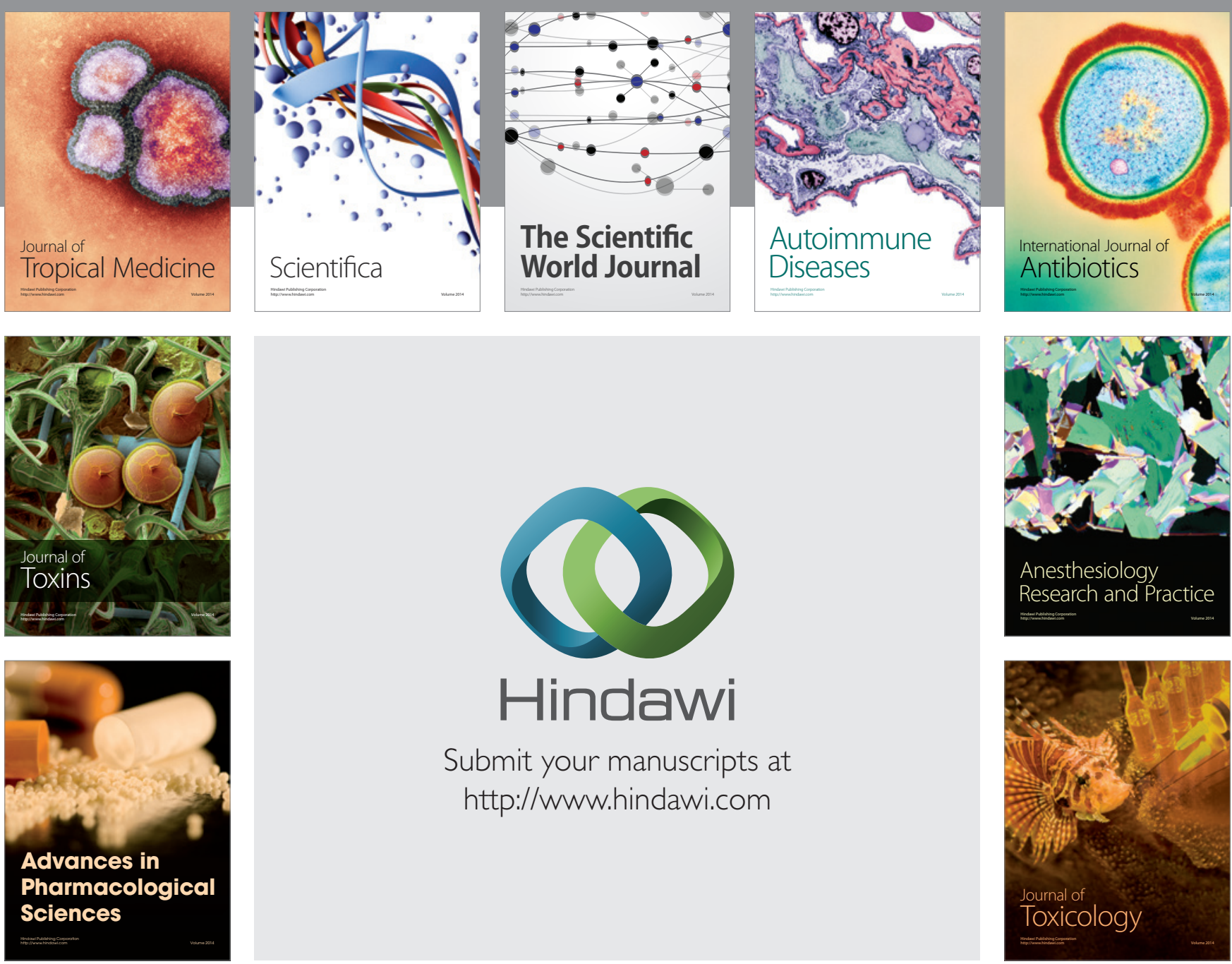

\section{Hindawi}

Submit your manuscripts at

http://www.hindawi.com
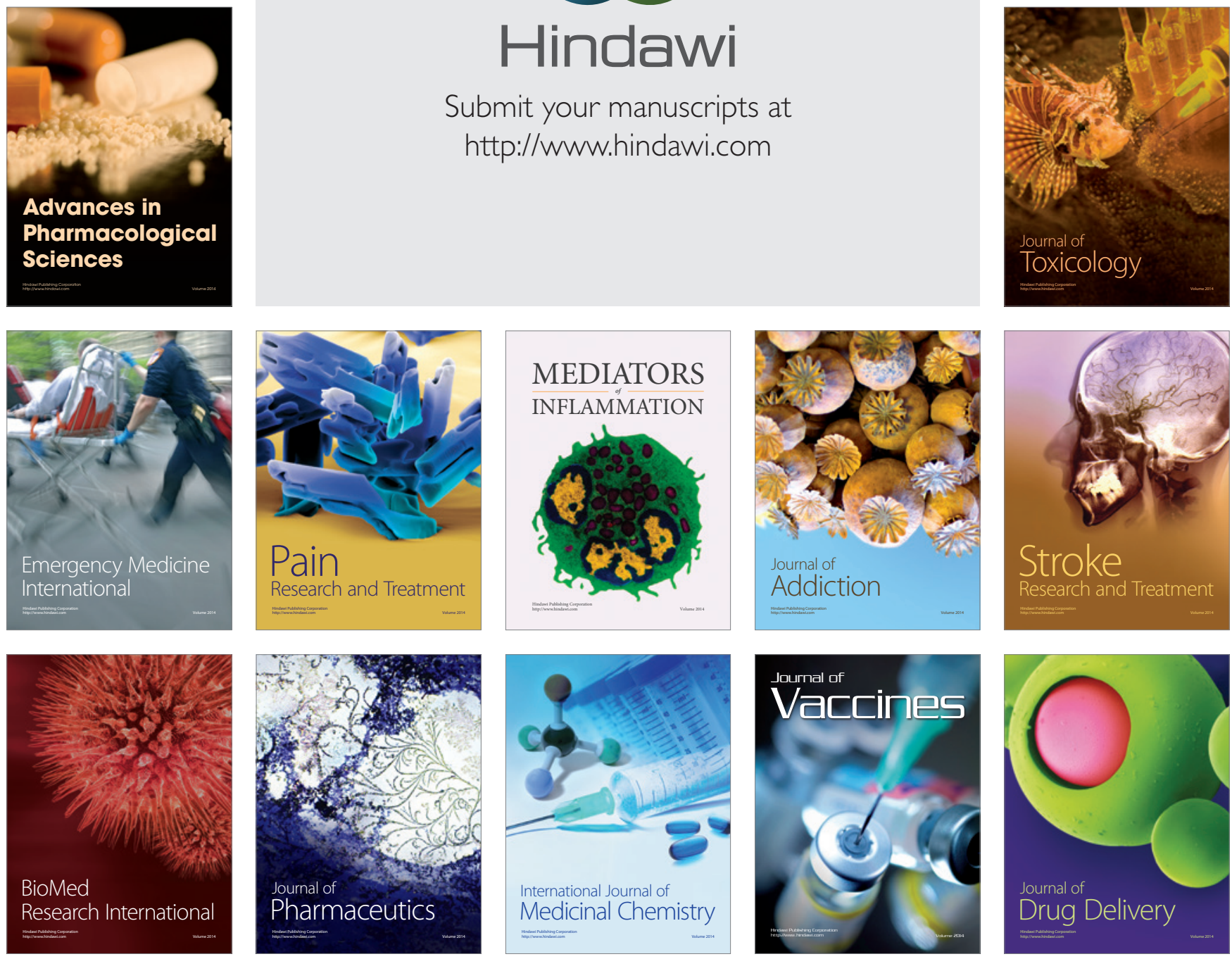